\title{
Contributo allo studio degli sviluppi in serie di polinomî di Laguerre.
}

Memoria di Ugo Brogqr (Roquebrune-Cap Martin-France A. M.).

Sunto. - La condizione $\mathrm{c}_{0}^{z}+\left(\mathrm{c}_{0}+\mathrm{c}_{1}\right)^{2}+\ldots<\infty$ è posta a fondamento dello studio degli sviluppi $\mathrm{e}_{0} \mathrm{~L}_{0}(\mathrm{t})+c_{1} \mathrm{~L}_{1}(\mathrm{t})+\ldots$ in serie di polinom $\hat{\imath}$ di Laguerre.

È nota l'importanza della condizione $c_{0}^{2}+c_{1}^{2}+. .<\infty$ per lo studio degli sviluppi $c_{0} \varphi_{0}(t)+c_{1} \varphi_{1}(t)+\ldots$ in serie di funzioni ortogonali $\varphi_{n}(t)$.

Se è $\varphi_{n}(t)=e^{-t / 2} L_{n}(t)$, e $L_{n}(t)=\frac{e^{t}}{n !} D^{\prime \prime}\left(e^{-t} t^{n}\right)$ è il polinomio di LaguerRe di ordine $n$, ne assume una forse non minore la condizione più restrittiva $e_{0}^{2}+\left(c_{0}+c_{1}\right)^{2}+\ldots<\infty$, sufficente per la convergenza della serie $\Sigma c_{n} L_{n}(t)$, $(t \geq 0)$, per la convergenza uniforme dell' altra $\Sigma\left(c_{n}+c_{1}+\ldots+c_{n}\right)\left\{L_{n,}(t)-L_{n+1}(t)\right\}$, $(0 \leq \alpha \leq t \leq \beta)$, perchè, se $\varphi(t) \sim \Sigma c_{n} L_{n}(t)$, sia $\varphi(t)=\Sigma c_{n,} L_{n}(t)=\Sigma\left(c_{0}+c_{1}+\ldots\right.$ $\left.\ldots+c_{n}\right)\left\{L_{n}(t)-L_{n+1}(t)\right\}$ in ogni punto dell intervallo $(<0, \infty)$, necessaria e sufficiente per l'esistenza della derivata $\frac{d \varphi(t)}{d t}$ di $\varphi(t)=\int_{0}^{t} \frac{d \varphi(t)}{d t} d t$, qualunque
sia $t>0$.

Questi risultati, oggetto dei primi tre paragrafi, sono suscettibili di una facile generalizzazione. Essi consentono inoltre di dare condizioni sufficenti di convergenza della serie $c_{0}^{2}+\left(c_{0}+c_{1}\right)^{2}+\ldots$, dove $c_{n}=a_{0} b_{n}+a_{1} b_{n-1}+\ldots+a_{n} b_{n}$, e, se queste sono soddisfatte, di dedurre dalla somma delle serie $\Sigma a_{n} L_{n}(t)$, $\Sigma b_{n} L_{n}(t)$, quella di $\Sigma c_{n} L_{n}(t)$.

La relazione ( $\left.{ }^{1}\right)$

$$
\int_{0}^{\infty} e^{-t s} L_{n}(t) d t=\frac{1}{s}\left(\frac{s-1}{s}\right)^{n} \quad(R(s)>0)
$$

che come si è dimostrato altrove $\left({ }^{2}\right)$, consente di dedurre nel modo più im.

(1) Cfr. F. Tricomi, Trasf. di Laplace e polinomî di Laguerre, "Rend. Lincei ", XXI, (1935), pp. 232-239.

(2) U. Brockr, Sui polinomi di Laguerre e una limitazione di Szegö, "Bollettino Un. Mat, It. », II, 1, (1939), pp. 213-217. 
mediato le proprietà fondamentali dei polinomî $L_{n}(t)$, fa corrispondere allo sviluppo della funzione

$$
s f(s)=s \int_{0}^{\infty} e^{-t s} \varphi(t) d t
$$

supposta regolare nel sempiano $R(s)>\frac{1}{2}$, in serie di potenze di $\frac{s-1}{s}$

$$
s f(s)=\sum_{n=0}^{\infty} c_{n}\left(\frac{s-1}{s}\right)^{n}, \quad\left(R(s)>\frac{1}{2}\right)
$$

lo sviluppo formale della funzione $\varphi(t)$ in serie di polinomï di LaGUERRE

$$
\varphi(t) \sim \sum_{n=0}^{\infty} c_{n} L_{n}(t)
$$

Il suo intervento nella deduzione dei risultati annunziati appare senz' altro chiarito da ciò che, se

$$
s f(s)=\int_{0}^{\infty} e^{-t s} \psi(t) d t, \quad\left(R(s)>\frac{1}{2}\right)
$$

è anche

$$
\begin{gathered}
\downarrow(t)=\frac{d \varphi(t)}{d t}, \\
s^{2} f(s)=\sum_{n=0}^{\infty}\left(c_{0}+c_{1}+\ldots+c_{n}\right)\left(\frac{s-1}{s}\right)^{n} .
\end{gathered}
$$

1. Poichè, come è noto, il sistema $\left\{e^{-t / 2} L_{n}(t)\right\}$ è ortogonale e normalizzato in $(0, \infty)$ :

a) $e^{-t / 2} \Sigma \Gamma_{n} L_{n}(t)$ è, se $\Gamma_{0}^{2}+\Gamma_{1}^{2}+\ldots<\infty$, lo sviluppo formale di una funzione di quadrato sommabile, (teorema di Fischer-RIEsz), e

b) se $\Gamma_{0}^{2}+\Gamma_{1}^{2}+\ldots<\infty$, la serie $\Sigma \Gamma_{n} \int_{0}^{t} e^{-u / 2} L_{n}(u) d u$ converge uniformemente in ogni intervallo chiuso $\langle 0, T\rangle\left(^{3}\right)$.

Ma è anche, come è ugualmente noto e come la (1) permette di vedere senz' altro

$$
L_{n}(t)=L_{n}^{\prime}(t)-L_{n+1}^{\prime}(t)
$$

(3) Cfr. ad es. Kaczmarz-Steinhaus, Theorie der Orthogonalreihen, (1935), p. 60. 
E quindi

$$
\int_{0}^{t} e^{-u t^{\prime} / 2} L_{n}(u) d u=2 e^{-t / 2}\left[L_{n}(t)-L_{n+1}(t)\right]-\int_{0}^{t} e^{-u_{1} / 2} L_{n+1}(u) d u
$$

e poichè la serie

$$
\sum_{n=0}^{\infty} \Gamma_{n} \int_{0}^{t} e^{-u / 2}\left[L_{n}(u)+L_{n+1}(u)\right] d u
$$

somma di due serie uniformemente convergenti, converge uniformenente, converge uniformemente l'altra

$$
\sum_{n=0}^{\infty} \Gamma_{n}\left[L_{n}(t)-L_{n+1}(t)\right]
$$

La convergenza della serie

$$
\underset{n=0}{\infty} c_{n} L_{n}(t)
$$

$\left(c_{n}=\Gamma_{n}-\Gamma_{n-1}\right)$, in corrispondenza di ogni $t \geq 0$, ̀̀ una conseguenza di quanto precede e del teorema secondo il quale $\Sigma a_{v} b_{v}$ converge, se

a) $\Sigma\left(a_{0}+a_{1}+\ldots+a_{\nu}\right)\left(b_{\nu}-b_{\nu+1}\right)$ converge;

B) se $\lim _{p \rightarrow \infty}\left(a_{0}+a_{1}+\ldots+a_{p}\right) b_{p+1}$ esiste ( $\left.{ }^{*}\right)$.

È infatti, se $t \geq 0$

$$
\left|L_{n}(t)\right| \leq e^{t / 2}\left({ }^{j}\right)
$$

e quindi, poichè $\lim _{p \rightarrow \infty}\left(c_{0}+c_{1}+\ldots+c_{p}\right)=\lim _{p \rightarrow \infty} \Gamma_{p}=0$

$$
\lim _{p \rightarrow \infty}\left(c_{0}+c_{1}+\ldots+c_{p}\right) L_{p+1}(t)=0 .
$$

Si ha dunque anche, per la formola di sommazione di Brunacci-ABEL

$$
\begin{aligned}
& \sum_{n=0}^{p} c_{n} L_{n}(t)=\sum_{n=1}^{p} \Gamma_{n}\left[L,(t)-L_{n+1}(t)\right]-\Gamma_{0} L_{1}(t)+\Gamma_{n+p} L_{n+p+1} \\
& \sum_{n=0}^{\infty} c_{n} L_{n}(t)=\sum_{n=0}^{\infty} \Gamma_{n}\left[L_{n}(t)-L_{n+1}(t)\right] .
\end{aligned}
$$

Si è cioè dimostrato che

I) $\ll$ Sia $\Gamma_{n}=c_{0}+c_{1}+\ldots+c_{n}, \Gamma_{0}^{2}+\Gamma_{1}^{2}+\ldots<\infty$.

(4) Cfr. ad es, O. KNopP, Th. und Anwendung der unendl. Reihen, (1922), pag. 305.

${ }^{(5)}$ Cfr. G. SzEGö, "Math. Zoitschrift », vol. 1, (1918), pagg. 341-352, \$ 2. 
La serie

$$
\underset{n=0}{\infty} C_{n} L_{n}(t)
$$

converge se $\mathrm{t} \geq 0$, la serie

$$
\sum_{n=0}^{\infty} \Gamma_{n}\left[L_{n}(t)-L_{n+1}(t)\right]
$$

converge uniformemente in ogni intervallo $0 \leq \alpha \leq \mathrm{t} \leq \beta$, ed $\grave{e}$

$$
\sum_{n=0}^{\infty} c_{n} L_{n}(t)=\sum_{n=0}^{\infty} \Gamma_{n}\left[L_{n}(t)-L_{n+1}(t)\right] » .
$$

3. II) $\ll$ Sia $\Gamma_{n}=c_{0}+c_{1}+\ldots+e_{n} ; p(t) \propto \sum_{n=0}^{\infty} e_{n} L_{n}(t)$.

La condizione

$$
\Gamma_{0}^{2}+\Gamma_{1}^{2}+\ldots<\infty
$$

$\grave{e}$ necessaria e sufficiente perchè in $(0, \infty)$ :

a) $c(t)$ sia assolutamente continua, e si abbia

$$
\varphi(t)=\int_{0}^{t} \frac{d \varphi(u)}{d u} d u
$$

B) $e^{-\mathrm{t} / 2} \varphi(\mathrm{t}), \mathrm{e}^{-\mathrm{t} / 2} \frac{\mathrm{d} \varphi(\mathrm{t})}{\mathrm{d} t}$ siano di quadrato sommabile ».

La condizione è necessaria.

Se $e^{-t / 2} \varphi(t)$ ̀̀ di quadrato sommabile e $\varphi(t) \sim \sum_{n=0}^{\infty} c_{n} L_{n}(t)$ è pure. poichè il sistema $\left\{e^{-t / 2} L_{n}(t)\right\}$ è completo,

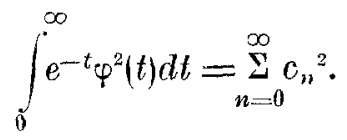

Si tratta di dimostrare che se $\frac{d \varphi(t)}{d t}$ esiste, e e $e^{-t / 2} \frac{d \varphi(t)}{d t}$ è cli quadrato sommabile, ̀̀

$$
\begin{gathered}
\frac{d \varphi(t)}{d t} \sim \stackrel{\Sigma}{n=0}_{\sum_{n}}^{\infty} \Gamma_{n} L_{n}(t), \quad\left(\Gamma_{n}=c_{n}+c_{1}+\ldots+c_{n}\right) \\
\int_{0}^{\infty} e^{-t}\left(\frac{d \varphi(t)}{d t}\right)^{2} d i=\sum_{n=0}^{\infty} \Gamma_{n}{ }^{2} .
\end{gathered}
$$


Le ascisse di convergenza delle trasformate di LAPLACE

$$
\begin{gathered}
f(s)=\int_{0}^{\infty} e^{-t s} \varphi(t) d t \\
F(s)=\int_{0}^{\infty} e^{-t s} \frac{d \varphi(t)}{d t} d t
\end{gathered}
$$

non sono maggiori di $1 / 2$.

Si ha infatti, per la disuguaglianza di ScHWARZ

$$
\left(\int_{0}^{\infty} e^{-t(s-1 / 2)} e^{t / 2}|\psi(t)| d t\right)^{2} \leq \int_{0}^{\infty} e^{-t(2 s-1)} d t \cdot \int_{0}^{\infty} e^{-t} \psi^{2}(t) d t .
$$

$s f(s)$, regolare nel semipiano $R s>\frac{1}{2}$, vi ammette lo sviluppo $\Sigma c^{\prime}{ }_{n}\left(\frac{s-1}{s}\right)^{n}$, mentre, poichè, come si vede integrando per parti,

può scriversi

$$
s f(s)=F(s),
$$

$$
\begin{aligned}
& s F(s)=s^{2} f(s)=s \sum_{\nu=0}^{\infty}\left(\frac{s-1}{s}\right)^{\nu} \sum_{\mu=0}^{\infty} c_{\mu}^{\prime}\left(\frac{s-1}{s}\right)^{\mu} \\
& =s \sum_{n=0}^{\infty}\left(c_{0}^{\prime}+c^{\prime}{ }_{1}+\ldots+c^{\prime}{ }_{n}\right)\left(\frac{s-1}{s}\right)^{n} \text {. }
\end{aligned}
$$

Risulta finalmente da

$$
\begin{gathered}
f(s)=\int_{0}^{\infty} e^{-t s} \varphi(t) d t=\frac{1}{s} \sum_{n=0}^{\infty} c^{\prime}{ }_{n}\left(\frac{s-1}{s}\right)^{n} \\
\varphi(t) \sim \sum_{n=0}^{\infty} c_{n}^{\prime} L_{n}(t) \\
c_{n}^{\prime}=c_{n}, \quad c_{0}^{\prime}+c_{1}^{\prime}+\ldots+c^{\prime}{ }_{n}=\Gamma_{n},
\end{gathered}
$$

e, poichè $e^{-t / 2} \frac{d \varphi(t)}{d t} \dot{e}$ di quadrato sommabile,

$$
\Gamma_{0}^{2}+\Gamma_{1}^{2}+\ldots<\infty \text {. }
$$

La condizione è sufficiente.

Il teorema di FIScher-RIEsz permette di scrivere, poichè

$$
\begin{gathered}
\Gamma_{0}^{2}+\Gamma_{1}^{*}+\ldots<-\infty \\
F(s)=\frac{1}{s} \sum_{n=0}^{\infty} \Gamma_{n}\left(\frac{s-1}{s}\right)^{n}=\int_{0}^{\infty} e^{-t s} \psi(t) d t, \quad\left(R s>\frac{1}{2}\right)
\end{gathered}
$$


dove, $(n=0,1, \ldots)$

$$
\Gamma_{n}=\int_{0}^{\infty} e^{-t} \psi(t) L_{n}(t) d t
$$

Dalla convergenza di $\Gamma_{0}^{2}+\Gamma_{1}^{2}+\ldots$ segue quella di $\Gamma_{0} \Gamma_{1}+\Gamma_{1} \Gamma_{2}+\ldots$ e pertanto anche di $\left(\Gamma_{0}-\Gamma_{1}\right)^{2}+\left(\Gamma_{1}-\Gamma_{2}\right)^{2}+\ldots=c_{0}^{2}+c_{1}^{2}+\ldots$, mentre

$$
\begin{aligned}
& \frac{1}{s} F(s)=\frac{1}{s}\left(1-\frac{s-1}{s}\right) \sum_{n=0}^{\infty} \Gamma_{n}\left(\frac{s-1}{s}\right)^{n} \\
& =\frac{1}{s} \underset{\Sigma_{n=0}^{\infty}}{\infty} c_{n}\left(\frac{s-1}{s}\right)^{n}=f(s)=\int_{0}^{\infty} e^{-t s} \int_{0}^{t} \psi(u) d u d t .
\end{aligned}
$$

È dunque, quasi dappertutto $\left({ }^{6}\right)$

$$
\begin{aligned}
& \varphi(t)=\int_{0}^{t} \psi(u) d u \\
& \psi(t)=\frac{d \varphi(t)}{d t} .
\end{aligned}
$$

3. III) $\ll S e \dot{e}$

è pure

$$
c_{n}=\int_{0}^{\infty} e^{-t} L_{n}(t) \varphi(t) d t \quad(n=0,1, \ldots)
$$

$$
c_{0}+c_{1}+\ldots+c_{n}=\Gamma_{n} ; \quad \Gamma_{0}^{2}+\Gamma_{1}^{2}+\ldots<\infty
$$

$$
\varphi(t)=\sum_{n=0}^{\infty} c_{n} L_{n}(t), \quad(t \geq 0) » .
$$

İ per ipotesi, $\mathrm{I}_{0}^{2}+\Gamma_{1}^{2}+\ldots<\infty$. La serie

$$
\sum_{n=0}^{\infty} \Gamma_{n}\left\{L_{n}(t)-L_{n+1}(t)\right\}
$$

converge uniformemente in ogni intervallo chinso finito $0 \leq \alpha \leq t \leq \beta$. Poichè

$$
\int_{0}^{\infty}\left|e^{-t} L_{m}(t)\right| d t
$$

converge, è lecito s' integxi

$$
\int_{0}^{\infty} e^{-t} L_{m}(t) \sum_{n=0}^{\infty} \Gamma_{n}\left[L_{n}(t)-L_{n+1}(t)\right] d t
$$

(5) Cfr. M. Lerch, « Acta Math. », 27, (1903), pagg. 339-351, 345; BrogGI ( $\left.{ }^{2}\right)$. 
termine a termine (7), e s'ottenga così come valore dell'integrale, e qualunque sia $m, c_{m}$.

Se $\varphi(t)$ è una qualsiasi funzione tale che

$$
\int_{0}^{\infty} e^{-t} L_{m}(t) \varphi(t) d t=c_{m} \quad(m=0,1, \ldots)
$$

e $\Gamma_{0}^{2}+\Gamma_{1}^{2}+\ldots$ converge, essa è, come $\Sigma \Gamma_{n}\left[L_{n}(t)-L_{n+1}(t)\right]$, assolutamente continua in $(0, \infty)$ : continua pertanto in corrispondenza di ogni $t$ positivo e finito.

Dalla chiusura del sistema $\left\{e^{-t / 2} L_{n}(t)\right\}\left(^{8}\right)$ e da

$$
\sum_{n=0}^{\infty} \Gamma_{n}\left[L_{n}(t)-L_{n+1}(t)\right]=\sum_{n=0}^{\infty} c_{n} L_{n}(t)
$$

si deduce, qualunque sia $t \geq 0$,

$$
\varphi(t)=\sum_{n=0}^{\infty} c_{n} L_{n}(t)
$$

come si affermò.

4. Se ̀े $\Delta^{\prime \prime} a_{n}=a_{n+r}-\left(\begin{array}{l}r \\ 1\end{array}\right) a_{n+,+-1}+\ldots+(-)^{r} a_{n} ; a_{-r}=a_{-r+1}=\ldots=a_{-1}=0$; è pure

$$
(-)^{r} \sum_{n=0}^{\infty} a_{n} \Delta^{r} b_{n} \sim \sum_{n=0}^{\infty} b_{n} \Delta^{r} a_{n-r}
$$

Si ha dunque da (2) e dai precedenti teoremi

IV) « Sia

$$
\begin{aligned}
& \Gamma_{-n}=\Gamma_{-h+1}=\ldots=\Gamma_{-1}=0 \\
& \varphi(t)=\underset{n=-n}{\infty} \Delta^{h} \Gamma_{n} \cdot L_{n+h}(t) .
\end{aligned}
$$

La condizione

$$
\Gamma_{0}^{2}+\Gamma_{1}^{2}+\ldots<\infty
$$

è necessaria e sufficiente perchè spetto $a \mathrm{t}$;

1. esistano quasi dappertutto in $(0, \infty)$ le prime $\mathrm{h}$ derivate di $\varphi(\mathrm{t}) \mathrm{ri}$ -

(7) Cfr. T. J. J'. Bromwich, An introduction to the Theory of infinite Series, (1926), pag. 499 .

(') Cfr. ad es. G. Vitali e G. Sansone, Moderna teoria delle funzioni di variabile reale, Vol. II, pagg. 208-210; BRoGGI ( $\left.{ }^{2}\right)$. 
2. $\operatorname{sia} \varphi(0)=\varphi^{\prime}(0)=\ldots=\varphi^{(\mathrm{h}-1)}(0)=0$;

3. $\mathrm{e}^{-\mathrm{t} / 2} \frac{\mathrm{d}^{\mathrm{s}} \varphi(\mathrm{t})}{\mathrm{d} \mathrm{t}^{\mathrm{s}}}$ sia di quadrato sommabile $(\mathrm{s}=0,1, \ldots, \mathrm{h})$ :

4. perchè sia in corrispondenza di ogni $\mathrm{t} \geq 0$

$$
\begin{aligned}
\varphi^{(s)}(t) & =\sum_{n=-h+s}^{\infty} \Delta^{n-s} \Gamma_{n} \cdot L_{\dot{n}+h-s}(t) \\
& =(-)^{h-s} \sum_{n=0}^{\infty} \Gamma_{n} \cdot \Delta^{h-s} L_{n}(t) . \quad(s=0,1, \ldots, h-1)
\end{aligned}
$$

La serie al secondo membro converge in ogni punto finito dell' intervallo $(0, \infty)$, la serie al terzo membro converge uniformemente in ogni intervallo $0 \leq \alpha \leq t \leq \beta$ ».

5. V) $\ll$ Sia

$$
\begin{gathered}
A_{n}=a_{0}+a_{1}+\ldots+a_{n} ; \quad B_{n}=b_{n}+b_{1}+\ldots+b_{n} ; \\
c_{n}=a_{n} b_{0}+a_{n-1} b_{1}+\ldots+a_{10} b_{n} ; \quad \Gamma_{n}=c_{0}+c_{1}+\ldots+c_{n} ;
\end{gathered}
$$

e convergano

$$
A_{0}^{2}+A_{1}^{2}+\ldots ; \quad B_{0}^{2}+B_{1}^{2}+\ldots ; \quad c_{0}^{2}+c_{1}^{2}+\ldots .
$$

Può affermarsi che

1. converge parimenti $\Gamma_{0}^{2}+\Gamma_{1}^{2}+\ldots$;

2. $s e \grave{e}$

$$
\varphi_{1}(t)=\sum_{n=0}^{\infty} a_{n} L_{n}(t) ; \quad \varphi_{2}(t)=\sum_{n=0}^{\infty} b_{n} L_{n}(t)
$$

̀̀ pure

$$
\int_{0}^{t} \frac{d \varphi_{1}(t-u)}{d t} \varphi_{2}(u) d u
$$

la comune somma delle due serie

$$
\sum_{n=0}^{\infty} c_{n} L_{n}(t) ; \quad \sum_{n=0}^{\infty} \Gamma_{n}\left[L_{n}(t)-L_{n+1}(t)\right.
$$

di cui la prima converge qualunque sia $\mathrm{t} \geq 0$, la seconda converge uniforme= mente in ogni intervallo $0 \leq \alpha \leq \mathrm{t} \leq \beta$ ».

Si dimostra V) osservando che, per la supposta convergenza di $A_{0}^{2}+A_{1}^{2}+\ldots$; $B_{0}^{2}+B_{1}^{2}+\ldots$, convergono le serie sviluppo di $\varphi_{1}(t), \varphi_{2}(t)$, esistono in $(0, \infty)$ le derivate prime di $\varphi_{1}(t), \varphi_{2}(t)$, ed $e^{-t / 2} \varphi_{1}(t), e^{-t / 2} \frac{d \varphi_{1}(t)}{d t}, e^{-t / 2} \varphi_{2}(t), e^{-t / 2} \frac{d \varphi_{2}(t)}{d t}$ sono 
di quadrato sommabile; le loro trasformate di LAPLACE $f_{4}(s), s f_{1}(s), f_{2}(s), s f_{2}(s)$ convergono nel semipiano $R(s)>\frac{1}{2}$.

Può dunque scriversi

$$
\begin{aligned}
& f_{\mathrm{i}}(s)=\int_{0}^{\infty} e^{-t s} \varphi_{1}(t) d t=\frac{1}{s} \sum_{n=0}^{\infty} \alpha_{n}\left(\frac{s-1}{s}\right)^{n}, \\
& f_{2}(s)=\int_{0}^{\infty} e^{-t s_{\varphi_{2}}}(t) d t=\frac{1}{s_{n=0}} \sum_{n}^{\infty} b_{n}\left(\frac{s-1}{s}\right)^{n} .
\end{aligned}
$$

Che sia poi anche

$$
\begin{array}{rlrl}
f_{1}(s) \cdot f_{2}(s) & =\frac{1}{s^{2}} \sum_{n=0}^{\infty}\left(a_{n} b_{0}+a_{n-1} b_{1}+\ldots+a_{0} b_{n}\right)\left(\frac{s-1}{s}\right)^{n}=\frac{1}{s^{2}} \sum_{n=0}^{\infty} c_{n}\left(\frac{s-1}{s}\right)^{n} \\
& =\int_{0}^{\infty} e^{-t s}\left(\int_{0}^{t} \varphi_{1}(t-u) \varphi_{2}(u) d u\right) d t & \left(R(s)>\frac{1}{2}\right)
\end{array}
$$

̀̀ una conseguenza del convergere di $s f_{1}(s), s f_{2}(s)$ in $R(s)>\frac{1}{2}$ : convergono assolutamente $\left({ }^{9}\right)$ in $R(s)>\frac{1}{2} f_{1}(s), f_{2}(s)$ e sussiste pertanto il teorema della « Faltung $»\left({ }^{10}\right)$.

Poichè, per ipotesi, $c_{v}^{2}+c_{1}^{2}+\ldots$ converge, converge parimenti in $R(s)>\frac{1}{2}$ l'integrale di LAPLACE

e vi è uguale a

$$
\int_{0}^{\infty} e^{-t s}\left(\frac{d}{d t} \int_{0}^{t} \varphi_{1}(t-u) \varphi_{2}(u) d u\right) d t
$$

$$
s f_{1}(s) f_{2}(s)=\frac{1}{s} \underset{n=0}{\sum} c_{n_{2}}\left(\frac{s-1}{s}\right)^{n}
$$

la funzione assolutamente continua $\int_{0}^{t} \varphi_{1}(t-u) \varphi_{2}(u) d u$ ha infatti quasi dappertutto una derivata in $t>0$ ed il limite 0 per $t \rightarrow+0$ ("').

(9) G. Döтsch, Theorie und Anwendung der Lapplace-Transformation, (1937), pag. 149. La dimostrazione vale invariata se gl'integrali esprimenti $s f_{1}(s)$, sf $f_{2}(s)$ sono integrali secondo Lebesgue.

(10) G. Dötsch, loc. eit., pag. 161, teorema $I V_{\mathrm{b}}$.

(11) G. Dötsch, Joc. cit., pag. 152, teorema I. 
Per il teorema II) $\varphi_{1}(t), \varphi_{2}(t)$ sono assolutamente continue in $(0, \infty)$ e limitate in ogni intervallo $(0, T)$.

$\frac{d \varphi_{1}(t)}{d t}$ esiste quasi dappertutto in $(0, \infty)$ ed è $\varphi_{1}(0)=\varphi_{2}(0)=0$. 亡̀ cioè

$$
\begin{aligned}
\frac{d}{d t} \int_{0}^{t} \varphi_{1}(t-u) \varphi_{2}(u) d u & =\varphi_{1}(0) \varphi_{2}(t)+\int_{0}^{t} \frac{d \varphi_{1}(t-u)}{d t} \varphi_{2}(u) d u \\
& =\int_{0}^{t} \frac{d \varphi_{1}(t-u)}{d t} \varphi_{2}(u) d u \\
s f_{1}(s) f_{2}(s) & =\int_{0}^{\infty} e^{-t s}\left(\int_{0}^{t} \frac{d \varphi_{1}(t-u)}{d t} \varphi_{2}(u) d u\right) d t .
\end{aligned}
$$

$\int_{0}^{t} \frac{d \varphi_{1}(t-u)}{d t} \varphi_{2}(u) d u$ è una funzione integrale, nulla se $t=0$, e tale che $e^{-t / 2} \int_{0}^{t} \frac{d \varphi_{1}(t-u)}{d t} \varphi_{2}(u) d u$ è di quadrato sommabile: per il teorema II)

$$
\Gamma_{0}^{2}+\Gamma_{1}^{2}+\ldots<\infty\left({ }^{12}\right),
$$

così come convergono, con somma

$$
\int_{0}^{t} \frac{d \varphi_{1}(t-u)}{d u} \varphi_{2}(u) d u
$$

$\sum_{n=0}^{\infty} c_{n} L_{n}(t)$, qualunque sia $t \geq 0$

$\sum_{n=0}^{\infty} \mathrm{T}_{n}\left[L_{n}(t)-L_{n+1}(t)\right]$, uniformemente in ogni intervallo $0 \leq \alpha \leq t \leq \beta$.

(12) Per il teorema II è in $R(s)>\frac{1}{2}$

$$
\begin{aligned}
s^{2} f_{1}(s) f_{2}(s) & =\frac{1}{s}{\underset{s n=0}{\infty} \Gamma_{n}\left(\frac{s-1}{s}\right)^{n}} \\
& =\int_{0}^{\infty} e^{-t s}\left(\frac{d}{d t} \int_{0}^{t} \frac{d \varphi_{1}(t-u)}{d t} \varphi_{2}(u) d u\right) d t=\int_{0}^{\infty} e^{-t s}\left(\frac{d}{d t} \int_{0}^{t} \varphi_{1}^{\prime}(u) \varphi_{2}(t-u) d u\right) d t .
\end{aligned}
$$

